\title{
Drug-induced acute liver disease
}

\author{
M.G. Lee, B. Hanchard and N.P. Williams
}

Departments of Medicine and Pathology, University Hospital, University of the West Indies, Kingston, Jamaica, W. Indies.

\begin{abstract}
Summary: Fifty three patients with drug-induced acute liver disease are reported. There were 35 females and 18 males with a mean age of 41 years. All but one patient had jaundice or hyperbilirubinaemia and 51 had abnormal liver enzymes. Histologically 38 patients $(72 \%)$ had cholestatic injury while 15 had cytotoxic parenchymal damage. Methyldopa, chlorpropamide, chlorpromazine, halothane and the contraceptive pill accounted for $60 \%$ of cases. Fifty one patients recovered after drug withdrawal while 2 died of hepatic failure. Drug-induced liver injury must be considered in patients presenting with evidence of hepatic disease as the majority will recover on withdrawal.
\end{abstract}

\section{Introduction}

Drug-induced liver disease is a common clinical problem. Up to $25 \%$ of cases of fulminant hepatic failure may be the result of adverse reactions to drugs. ${ }^{1,2}$ For certain drugs such as isoniazid, chlorpromazine and methyldopa minor abnormalities in liver function tests can be detected in up to $20 \%$ of asymptomatic patients during therapy. ${ }^{3}$ These patients have minimal nonspecific focal hepatitis on liver biopsy often associated with both portal and intralobular inflammation. In addition it is estimated that approximately $2 \%$ of jaundiced patients admitted to general hospitals have drug-induced liver disease. ${ }^{4}$ In geriatric facilities the incidence may be 10 -fold higher. ${ }^{1}$

The susceptibility of the liver to damage by drugs is a consequence of its primary role in drug metabolism. ${ }^{5}$ As the vast array of chemicals to which we are exposed expands and the number of implicated agents continue to increase, awareness of drug-induced liver disease is important in all areas of clinical medicine. Recognition of a drug aetiology is essential since the prognosis is generally good upon withdrawal.

We report on the clinical, aetiological and histological features of acute drug-induced liver disease seen at the University Hospital, Jamaica, over a 15 year period.

\section{Patients and methods}

Patients admitted to the University Hospital, Kingston, Jamaica with drug-induced liver disease in the

Correspondence: M.G. Lee, D.M., F.R.C.P. (C), Department of Medicine, University of the West Indies, Kingston 7, Jamaica, W.I.

Accepted: 25 January 1989
15 year period June 1973 to June 1988 were reviewed.

The clinical presenting features, biochemical abnormalities, drug intake and outcome were studied. The histological details of all liver biopsy specimens were also reviewed. The histological classification used is modified from that proposed by Zimmerman and Ishak, ${ }^{6}$ for drug-induced hepatic injury. The biochemical abnormalities recorded were the initial or the highest value obtained.

Criteria for inclusion in the study were: (1) presence of jaundice and/or abnormal liver-related liver enzymes temporally related to (2) recent drug exposure, (3) absence of known previous underlying liver disease, (4) compatible hepatic histological findings and (5) improvement of abnormal clinical and biochemical parameters on withdrawal of the offending drug.

Patients with alcoholic liver disease, viral hepatitis, gall stones or evidence of extrahepatic biliary obstruction were excluded.

\section{Results}

There were 53 patients with drug-induced hepatic damage who satisfied at least four of the above criteria. The mean age at presentation was 41 years (median, 40 years; range 14-85). There were 35 females with a mean age of 40 years (range 14-85) and 18 males with a mean age of 41 years (range $15-73)$. The age distributions were: $0-9$ years $(0 \%)$; $10-19$ years (13\%); $20-29(17 \%) ; 30-39(19 \%)$; $40-49$ (22\%); and 50 years and older $(29 \%)$.

The presenting clinical features are shown in Table I. All but one patient had jaundice or hyperbilirubinaemia (range $21-610 \mu \mathrm{mol} / \mathrm{l}$ ). Twenty one patients $(39 \%)$ had dark urine, and $8(15 \%)$ had fever

(C) The Fellowship of Postgraduate Medicine, 1989 
Table I Clinical presentation in 53 patients with drug related liver disease

\begin{tabular}{lcc}
\hline Symptom/sign & Number of patients & $(\%)$ \\
\hline Jaundice & 52 & $(98)$ \\
Dark urine & 21 & $(39)$ \\
Fever & 8 & $(15)$ \\
Pale stools & 8 & $(15)$ \\
Pruritus & 8 & $(15)$ \\
Pain (right upper abdomen) & 2 & $(4)$ \\
Hepatomegaly & 20 & $(38)$ \\
Skin rash & 8 & $(15)$ \\
\hline
\end{tabular}

and skin rash. The mean duration of symptoms was 35 days. Twenty patients $(38 \%)$ had clinical evidence of hepatomegaly $(1-9 \mathrm{~cm}$ below the right costal margin).

The liver-related enzymes (serum transaminase, alkaline phosphatase and gamma glutamyl transpeptidase) were abnormally elevated in the 51 patients in whom data was available. In 19 patients the abnormality was predominantly elevation of alkaline phosphatase (AP), gamma glutamyl transpeptidase (GGTP), and direct hyperbilirubinaemia. In 10 patients there was significant elevation of the transaminase with only minimal elevation of AP and GGTP. In 22 there was elevation of the transaminase, AP, GGTP and mixed hyperbilirubinaemia.

In $23 \%$ of patients there was elevation of the total leucocyte count (range 10.5-21.1 $\times 10^{9}$ cells/l. Five patients had absolute peripheral eosinophilia $(>7 / 1)$.

Based on the predominant histological pattern, the types of liver damage are shown in Table II. Cholestatic hepatic injury was the commonest injury observed, occurring in 38 patients $(72 \%)$. In 27 of these the changes were those of hepatocanalicular cholestasis (cholestasis with portal tract inflammation) and in 11 canalicular (bland) cholestasis was seen. Of the 15 cases with acute cytotoxic parenchymal injury, diffuse necrosis (as in viral hepatitis) was present in 10 , while submassive or massive necrosis was the predominant change seen in 4 . One patient had predominant steatosis.

Twenty two drugs were implicated in inducing liver injury (Table III). However, methyldopa (8), chlorpropamide (8), chlorpromazine (7), halothane (5) and the oral contraceptive pill (4) accounted for $60 \%$ of the cases. The type of injury produced was not predictable, but certain drugs were more often associated with a particular type of injury. Methyldopa was the causative agent in 8 patients. Of these hepatocanalicular cholestasis was seen in 5, predominant diffuse necrosis was seen in two and submassive hepatocellular necrosis in the other patient. Chlorpropamide produced hepatocanalicular cholestasis in 5 cases and diffuse necrosis in 3. Chlorp-
Table II Histological type of liver damage observed in 53 patients with drug related liver disease

Histological type Number

Cytotoxic

(A) Diffuse necrosis

(B) Submassive/massive necrosis

(C) Steatosis

Cholestatic

(A) Hepatocanalicular

(B) Canalicular
4

27

romazine produced hepatocanalicular cholestasis in $6 \overrightarrow{\vec{\omega}}$ and diffuse necrosis in 1 . The oral contraceptive pill was associated only with canalicular cholestatic 6 injury, while halothane produced both cytotoxic and $\underline{3}$ cholestatic damage.

Two patients died of hepatic failure. Both had massive hepatic necrosis induced by phenytoin and $\stackrel{\varnothing}{\oplus}$ chlorpropamide respectively. The remaining 51 patients $\omega$ recovered clinically and biochemically after a mean interval of 63 days (range 9-168 days).

\section{Discussion}

Hepatic injury of many types may result from com monly used and generally well tolerated therapeutit agents. This has been demonstrated in our study, as a variety of therapeutic agents produced several types of hepatic injury. In our patients methyldopa, chlorprop- $\frac{\circ}{\varnothing}$ amide, chlorpromazine, halothane and the oral cont- $\stackrel{2}{\rightarrow}$ raceptive pill were the most commonly implicated $\vec{O}$ drugs. Within the total number of patients with drug-induced liver injury there is an extremely variable frequency of injury from different drugs. However, only a minority of drugs known to cause abnormal hepatic function or jaundice account for the majority of reported cases. ${ }^{7}$ Apart from the contraceptive pill, 3 which uniformly produced predominant cholestasis, the other drugs produced varying reactions.

The most frequently recognized drug-induced hepatic reactions are asymptomatic increases in the serum amino-transferases. Therefore the reported $\frac{D}{O}$ clinical cases probably represent only a fraction of all drug-induced liver injury. In addition, drugs can cause $\widetilde{N}$ toxic effects on the liver which can mimic almost any $N$ naturally occurring acute or chronic hepatic disease in $N$ man. ${ }^{8}$ Occasionally therapeutic agents produce a $\stackrel{\omega}{0}$ symptomatic acute illness indistinguishable from viral hepatitis. ${ }^{9-11}$

Although absolute histological criteria of drug- $\mathbb{D}$ induced injury have not been established, liver biopsy plays a vital role in diagnosis. ${ }^{12}$ Primarily, biopsy is 
Table III Morphological injury and associated drugs

Cytotoxic

\section{Massive/submassive} necrosis

methyldopa

halothane

phenytoin

\section{Diffuse}

necrosis (Hepatitis)

Steatosis

Hepatocanalicular

Cholestatic

methyldopa

halothane

hydrochlorothiazide

chlorpropamide

chlorpromazine

amoxycillin

gold

phenobarbitone

isoniazid

\section{methyldopa}

halothane

phenytoin

chlorpropamide

chlorpromazine

penicillin

ocp

ampicillin

reserpine

diazepam

amitriptyline

triazolam

azathioprine

phenylbutazone

sulfasalazine

ocp - oral contraceptive pill

used to exclude the possibility of underlying chronic liver disease. In many instances chronic liver disease runs an insidious asymptomatic course similar to what is seen in some cases of drug-induced liver injury. ${ }^{3}$ Furthermore, in some cases jaundice may be the presenting feature and biopsy is the only reliable means of making an early diagnosis. ${ }^{13}$ When liver injury develops during treatment with an essential drug, liver biopsy plays an essential role in establishing a drug-induced injury or an unrelated disorder. ${ }^{12}$ Liver biopsy also provides information about the severity and prognosis in patients with drug-induced injury.

There are broad histological categories that relate to the morphology of drug-induced liver injury. Acute injury may be either cholestatic or cytotoxic, the latter manifesting virtually any of the morphological lesions known in hepatology. Diffuse massive or zonal necrosis may occur, accompanied by degeneration and steatosis. Cholestatic injury may be either canalicular (bland) or hepatocanalicular, when there is associated portal tract inflammation. ${ }^{6}$

Accordingly, the whole spectrum of acute and chronic hepatocellular injury may be reproduced by drugs. Nevertheless, there are certain histological features which invariably point to drug-related injury. Linear or patchy, well defined necrosis, abundant eosinophils, large mononuclear phagocytes, bile duct injury, steatosis and non-caseating granulomas are frequent accompaniments to the general histological picture. However, there is significant overlap between these reactions and those produced by viral hepatitis and mechanical large duct biliary tract obstruction. ${ }^{14}$

Final proof of an association between a drug and liver injury can only be established by epidemiological studies and rechallenge in an individual patient. However, in only a very few instances have patients been rechallenged with a drug. ${ }^{15}$ Death has been reported after rechallenge or when the drug was continued in the presence of symptoms. ${ }^{9}$ Rechallenge is therefore ethically unjustifiable. ${ }^{8}$ Consequently, validation of drug-induced hepatotoxicity depends on historical, clinical, biochemical and pathological data.$^{16}$ In our patients there was no history of previous or underlying liver disease, the hepatic abnormality developed in temporal relationship to drug administration and in the majority complete clinical and biochemical recovery occurred on drug withdrawal.

Seventy two per cent of patients in this series had a predominant cholestatic reaction. This is in keeping with a compilation of adverse effects of drugs on the liver which revealed that cholestatic drug reactions were much more common than other manifestations such as hepatic necrosis. ${ }^{17}$ In our patients the biochemical results did not correlate with the histological pattern in some patients. This indicates the unreliability of routine serum liver related enzymes in indicating a specific histological pattern. The serum enzymes are indirect tests which are useful in the initial screening and as a crude guide to the possible hepatic pattern of injury. They may also serve as a transition to more specific diagnostic tests and as markers to be followed over long periods of time. ${ }^{18}$

A number of factors affect susceptibility to druginduced hepatic injury. However, the mechanisms by which susceptibility is increased are poorly understood. A female predominance of drug-induced injury is reported by several authors. ${ }^{8,9,18,19}$ Age may be another factor as drug-induced liver injury tends to occur in 
older patients. ${ }^{4}$ In the present series, there was no patient under 9 years, and the number of patients increased with age, with the majority being women.

Drug reactions may be predictable (dose dependent) or unpredictable (dose independent). The majority of drug reactions fall into the latter category. ${ }^{3}$ The mechanism of drug-induced injury is uncertain but various mechanisms including hypersensitivity and genetic or acquired metabolic variation have been invoked. ${ }^{12}$ Certain drug reactions are accompanied by features that suggest the involvement of immunological mechanisms. These include fever, skin rashes, arthralgia, eosinophilia and autoantibodies. It is thought that in most instances, toxic drug

\section{References}

1. Rubin, E. Iatrogenic hepatic injury. Hum Pathol 1980, 11: 312-331.

2. Zimmerman, H.J. \& Maddrey, W.C. Toxic and drug induced hepatitis. In: Schiff, L. \& Schiff, E. (eds) Diseases of the Liver, 5th edition. J.B. Lippincott Co, Philadelphia, 1982, pp. 621-692.

3. Davis, M. Adverse effects of drugs on the liver. In: Jewell, D.P. \& Ireland, A. (eds) Topics in Gastroenterology, Vol. 14. Blackwell Scientific Publications, Oxford, 1986, pp. $231-250$.

4. Ockner, R.K. Drug induced liver disease. In: Zakim, D. \& Boyer, T.D. (eds) Hepatology. A Textbook of Liver Disease. W.B. Saunders Co, Philadelphia, 1982, pp. 691-722.

5. Mitchell, J.R. \& Jollows, D.J. Metabolic activation of drugs to toxic substances. Gastroenterology 1975, 68: $392-410$.

6. Zimmerman, H.J. \& Ishak, K.G. Hepatic injury due to drugs and toxins. In: MacSween, R.N.M., Anthony, P.P. \& Scheuer, P.J. (eds) Pathology of the Liver. Churchill Livingstone, Edinburgh, 1987, pp. 503-573.

7. Read, A.E. The liver and drugs. In: Wright, R., Alberti, K.G.M.M. \& Millward-Sadler, G.H. (eds) Liver and Biliary Disease. W.B. Saunders, London, 1979, pp. 822-847.

8. Sherlock, S. The spectrum of hepatotoxicity due to drugs. Lancet 1986, i: 440-444.

9. Rodman, J.S., Deutsch, D.J. \& Gutman, S.I. Methyldopa hepatitis. A report of six cases and review of the literature. Am J Med 1976, 60: 941-948.

10. Mitchell, J.R., Zimmerman, H.J., Ishak, K.G. et al. Isoniazid liver injury: clinical spectrum, pathology and probable pathogenesis. Ann Int Med 1976, 84: 181-192. metabolites or the immune responses to these $\frac{0}{0}$ metabolites mediate the damage. ${ }^{5}$ Selective impair-气 ment of certain vital cellular functions may result in 3 lethal injury to hepatocytes, whereas selective impair- $\stackrel{\mathbb{R}}{\Omega}$ ment to the bile secretory apparatus may result in. cholestasis. $^{20}$

In conclusion, the physician must consider a drug $\stackrel{\overline{\vec{S}}}{\stackrel{5}{+}}$ related aetiology in patients presenting with evidence of hepatic disease whatever the clinical picture. This is $\frac{\bar{\sigma}}{\bar{s}}$ especially important in middle aged or elderly $\vec{\sigma}$ patients. In such situations it is imperative to obtain a $\propto$ detailed and accurate drug history as discontinuation $\%$ of the offending drug will lead to improvement in the $\vec{\circ}$ majority.

11. Maddrey, W.C. \& Boitnott, J.K. Drug induced chronic 0 liver disease. Gastroenterology 1977, 72. $1348-1353$

12. International Group Review. Guidelines for diagnosis of therapeutic drug induced liver injury in liver biopsies. Lancet 1974, i: 854-857.

13. Mistilis, S.P. \& Blackburn, C.R.B. Active chronic음 hepatitis. Am J Med 1970, 48: 484-495.

14. Phillips, M.J. \& Poucell, S. Modern aspects of thec morphology of viral hepatitis. Hum Pathol 1981, 12: 1060-1084.

15. Ludwig, J. Drug effects on the liver. A tabular compila- $\overrightarrow{0}$ tion of drugs and drug related hepatic diseases. Dig Bs Sci 1979, 24: 785-796.

16. Ishak, K.G. \& Irey, N.S. Hepatic injury associated wî̀ the phenothiazines. Clinicopathologic and follow up study of 36 patients. Arch Pathol 1972, 93: 283-304.

17. Ludwig, J. Drug effects on the liver. An updated tabular compilation of drugs and drug related hepatic diseases. Dig Dis Sci 1983, 28: 651-666.

18. Kaplowitz, N., Eberle, D. \& Yamada, T. Biochemical tests for liver disease. In: Zakim, D. \& Boyer, T.D. (eds) Hepatology. A Textbook of Liver Disease. W.B. Saunders Co, Philadelphia, 1982, pp. 583-612.

19. Maddrey, W.C. Drug related acute and chronic hepatitis. Clin Gastroenterol 1980, 9: 213-224.

20. Kaplowitz, N., Aw, T.Y., Simon, F.R. et al. Drug induced hepatotoxicity. Ann Int Med 1986, 104:3. $826-839$. 